\title{
Elizabethkingia Meningoseptica: An Emerging Pathogen in Pediatric Patients.
}

\author{
Shaista Nazir, M.D. ', Dekyong Angmo, M.D. ${ }^{2}$, Bashir Ahmad Fomda, M.D. ${ }^{3}$, Javid lqbal, M.D. ${ }^{4}$ \\ 1.2 Senior Resident, ${ }^{3}$ Professor, Department of Microbiology ${ }^{4}$, Assistant Professor, Department of \\ Pediatrics, SKIMS, Soura, Srinagar
}

\section{A B S T R A C T}

E. meningoseptica is widely distributed in fresh and salt waters, in the soil, and in some animals but is not considered a normal human microflora. It is a gram negative non-fermentative bacillus which is found in the hospital environment and associated with nosocomial infections. It causes various disease like endocarditis, peritonitis, bacteremia, pneumonia, pediatric meningitis. It is mostly associated with infections in the immunocompromised patients. Recognition of $E$. meningoseptica is of paramount importance for clinicians since multi-drug resistance is common for this organism and secondly it is resistant to antibiotics which are normally used in the treatment of gram negative infections and is sensitive to vancomyin. So knowledge about this organism and its sensitivity will help clinicans to start the antibiotic of choice promptly. We here reported 2 cases of Elizabethkingia meningoseptica in blood and pleural fluid from immunocompromised pediatric patients. JMS 2014; 17(2):61-63

Key Words: Elizabethkingia meningoseptica, Chryseobacterium meningosepticum

Elizabethkingia was first discovered in 1959 by Elizabeth

O. King. ${ }^{1}$ King was an American bacteriologist who was working on an unclassified bacteria associated with meningitis in infants. Elizabethkingia meningoseptica previously known as Chryseobacterium meningosepticum is a gram-negative bacillus that is widely distributed in nature ${ }^{1}$. The genus Elizabethkingia has four species Elizabethkingia anophelis, Elizabethkingia meningoseptica, Elizabethkingia endophytica, and Elizabethkingia miricola.

Potential risk factors for developing E. meningoseptica bacteremia identified in the literature include malignancies, steroid use, diabetes mellitus, neutropenia, and organ transplant. ${ }^{2,3}$ Patients with multiple comorbid conditions (especially cancer, diabetes mellitus, chronic kidney disease or end-stage renal disease being treated with dialysis, alcoholism, alcoholic cirrhosis, immunocompromising conditions, immunosuppressive treatment) should prompt a high index of suspicion for Elizabethkingia infections. Elizabethkingia is typically

\section{Correspondence}

Prof Bashir Ahmad Fomda, M.D.

Department of Microbiology SKIMS, Srinagar. 190011. Email bashirfomda@gmail.com resistant to antibiotics used to treat gram-negative infections, including extended-spectrum beta-lactams, aminoglycosides, tetracycline, and chloramphenicol. Vancomycin and other alternatives include ciprofloxacin, minocycline, trimethoprim-sulfamethoxazole, rifampin, and novobiocin. ${ }^{4}$

It is a nonfastidious, glucose-nonfermenting, gram negative bacilli that are oxidase positive and nonmotile. They do not form spores and require oxygen for survival. They grow well on blood agar and chocolate agar. Colonies are very pale yellow and may not be easily evident at 24 hours. They are catalase positive, oxidase positive, indole positive, negative by nitrate reductase although some strains can be positive. ${ }^{5}$ They show greyish discolouration around the colonies on blood agar due to proteases and gelatinase. E. meningoseptica grow poorly on MacConkey agar and are considered glucose oxidizers. ${ }^{6}$ It is usually resistant to many antibiotics commonly used to treat infections caused by gram-negative bacteria. It is generally resistant to aminoglycosides, and $\beta$-lactam agents, including carbapenems, due to the production of extended spectrum $\beta$-lactamases (ESBL) and metallo- $\beta$-lactamases ${ }^{7}$.

In contrast, E. meningoseptica is often susceptible to agents 
used for gram-positive bacteria. Vancomycin has been described as an active agent against $E$. meningoseptica ${ }^{8,9}$

We reported 2 cases of Elizabethkingia meningoseptica in a patient with bacteremia and pyrexia of unknown origin.

\section{Case 1:}

A 9 year old male child known case of T-cell ALL treated with BFM 95 ALL protocol presented with relapse of T cell ALL. At the time of relapse child presented with fever and easy fatigability. On physical examination he had pallor. Laboratory investigations showed, $\mathrm{Hb}-9 \mathrm{~g} / \mathrm{dl}$, TLC40,000cells/ul, platelet-50000cells/ul. Manual DLC showing $80 \%$ blasts. On Bone marrow examination it was replaced by blasts $(98 \%)$. These blasts showed block positivity for PAS(periodic acid schiff) and negativity for MPO (myeloperoxidase) and SBB (sudan black B). Immunophenotyping was consistent with T cell ALL. Coagulogram and X-ray chest were normal. Patient was started on UK-ALL 12 protocol. Meanwhile, CBC was repeated which showed, $\mathrm{Hb}$ of $7 \mathrm{~g} / \mathrm{dl}$, TLC- 700 cells/ul, platelet-30000cells/ul. Patient blood culture was send as he was having fever and absolute neutrophil count of 200 cells $/ \mu 1$.

Blood culture bottles were processed in BacT/ALERT 3D system. A Gram smear was made from the flag positive bottle which showed gram negative bacilli. It was subcultured on blood agar and MacConkey agar. Growth of 1-2 mm colonies were seen on blood agar. There was no growth on MacConkey agar. Based on the growth characteristics, biochemical reactions, drug susceptibility pattern, and identification by Vitek 2 (Biomerieux) an automated microbial identification system the isolate was identified as E. meningoseptica. It was sensitive to vancomycin and resistant to colistin. The child was treated with IV vancomycin. He responded to the treatment and was discharged. He is now on follow up in the OPD with TLC 1500cells/ul , Hb 8g/dl, platelet-11000cells $/ \mu 1$.

\section{Case 2:}

A 2 yr old male child, product of non consangious marriage was admitted with history of high grade fever and cough since 1 week. On examination, patient was irritable with temperature of $38.5^{\circ} \mathrm{C}$, respiratory rate of $58 /$ minute with mild intercostal retraction, pulse 124 /minute, $\mathrm{SPO}_{2}$ of $93 \%$. On auscultation, air entry was decreased on right side, crepts were heard in right lower lobe of chest. An impression of community acquired Pneumonia was made. Patient was put on $\mathrm{O}_{2}$ inhalation via face mask and was put on empirical i.v ampicillin.

On lab evaluation $\mathrm{CBC}$ showed $\mathrm{Hb}$ of $10 \mathrm{gm} / \mathrm{dl}$, TLC$3500 /$ ul, neutrophils $58 \%$, lymphocytes $40 \%$, platelet of 150000. Chest X-ray showed right pleural effusion with underlying consolidation. Pleurocentesis was done for pleural fluid analysis which revealed exudative nature of the fluid. Pleural fluid was also sent for gram stain and culture. On gram stain, inflammatory cells were seen with no microorganism.

Culture of pleural fluid was done by inoculating it in liquoid broth and incubating at $37^{\circ} \mathrm{C}$. After overnight incubation, turbidity was seen and subculture was done on blood agar and MacConkey agar. The blood agar demonstrated growth of multiple yellow pigmented colonies. The organism was nonmotile, oxidase and indole positive. No growth was seen on MacConkey agar. It was identified as E. meningoseptica based on further biochemical reactions and automated bacteriology identification system (Vitek 2 system) in the laboratory. The isolate sensitivity was done on Mueller Hinton agar by the Kirby beaur disc diffusion method and it was resistant to colistin and sensitive to vancomycin.

Treatment of patient was changed from ampicillin to vancomycin . Patient responded to the treatment and improved clinically. Patient was later discharged and was on follow up in the out patient department.

\section{DISCUSSION}

E. meningoseptica is ubiquitously found in hospital environments. E. meningoseptica is most commonly associated with meningitis and a variety of infections in premature infants, newborns and immunocompromised individuals. Potential risk factors for developing $E$. meningoseptica bacteremia identified in the literature include malignancies, steroid use, diabetes mellitus, neutropenia, and organ transplant ${ }^{3}$. This bacterium proliferates in the hospital environment, growing on moist surfaces such as sinks, water tanks, ventilator tubing, saline solution use for flushing devices, etc., thus making it an emerging hospital-acquired pathogen. If isolated from clinical specimens, its pathogenic potential associated with clinical significance should be thoroughly evaluated. Since, growth of this organism was isolated from pediatric patients 
with history of malignancy and pyrexia of unknown origin, it is important to keep this infection as a differential diagnosis while treating such patients.

It should always be considered in the etiological diagnosis of septicemia, which is essential on clinical cases similar to those described in this article, in long-stay hospital patients with comorbidities, who are not responsive to empirical therapy and do not have a microbiological diagnosis defined. This results in an inappropriate choice of antimicrobial therapy, which may lead to negative consequences on the morbidity and mortality of patients infected by this pathogen.

Rapid, accurate identification of the strain may be challenging for clinical microbiology laboratories therefore a rapid automated identification is needed for its identification. Accurate identification of these strains by either complete biochemical investigation or updated automated identification systems is crucial in selecting appropriate antimicrobial susceptibility testing and proper antibiotic therapy.

Optimal antimicrobial guidelines to treat $E$. meningoseptica remain to be established. Infection with $E$. meningoseptica is clinically important as the organism is intrinsically resistant to multiple antibiotics, such as beta-lactams, aminoglycosides, tetracycline, tigecycline, colistin, chloramphenicol and carbapenems.. However, it is susceptible to the agents used to treat Gram-positive bacteria: rifampicin, ciprofloxacin, vancomycin and trimethoprim sulfamethoxazole.

Currently, validated susceptibility testing methods are not available and MIC breakpoints have not been established by the Clinical and Laboratory Standards Institute (CLSI) for E. meningoseptica. Results of susceptibility testing may vary substantially when different methods are used. In general, broth microdilution methodology is more reliable than disk diffusion technique

\section{REFERENCES}

1. Kim KK, Kim MK, Lim JH, Park HY, Lee S-T. Transfer of Chryseobacterium meningosepticum and Chryseobacterium miricola to Elizabethkingia gen. nov. as Elizabethkingia meningoseptica comb. nov. and Elizabethkingia miricola comb. nov. Int J Syst Evol Microbiol. 2005 May;55(Pt 3):128-793.

2. Hsu M-S, Liao C-H, Huang Y-T, Liu C-Y, Yang C-J, Kao $\mathrm{K}-\mathrm{L}$, et al. Clinical features, antimicrobial susceptibilities, and outcomes of Elizabethkingia meningoseptica (Chryseobacterium meningosepticum) bacteremia at a medical center in Taiwan, 1999-2006. Eur J Clin Microbiol Infect Dis. 2011 Oct; 30(10):12718.

3. Bloch KC, Nadarajah R, Jacobs R. Chryseobacterium meningosepticum: an emerging pathogen among immunocompromised adults. Report of 6 cases and literature review. Medicine (Baltimore). 1997 Jan;76(1):30-41.

4. Chiu C H; Waddingdon M; Hsieh W S; Greenberg D; Schreckenberger P C; Carnahan A M. Atypical Chryseobacterium meningosepticum and meningitis and sepsis in newborns and the immunocompromised, Taiwan. Emerg Infect Dis.2000 Sep-Oct; 6 (5): 481-486.

5. Lin P-Y, Chu C, Su L-H, Huang C-T, Chang W-Y, Chiu $\mathrm{C}-\mathrm{H}$. Clinical and Microbiological Analysis of Bloodstream Infections Caused by Chryseobacterium meningosepticum in Nonneonatal Patients. J Clin Microbiol, $2004 \mathrm{Jul} ; 42$ (7):33535.

6. H. Ratner. Flavobacterium meningosepticum. Infect Control, 1984 May; 5 (5): 237-239.

7. Di Pentima M C, Mason E.O. Jr, Kaplan S.L. In vitro antibiotic synergy against Flavobacterium meningosepticum: implications for therapeutic options .Clin Infect Dis, 1998 May;26 (5):1169-76

8. King EO. Studies on a group of previously unclassified bacteria associated with meningitis in infants. Am J Clin Pathol 1959 Mar;31(3):2417.

9. Lee SW, Tsai CA, Lee BJ. Chryseobacterium meningosepticum sepsis complicated with retroperitoneal hematoma and pleural effusion in a diabetic patient. J Chin Med Assoc 2008; 71(9):4736. 